\title{
Phenotype of tumor lymphatic vessels is a prognostic factor in human tongue squamous cell carcinoma
}

\author{
YUKIE NITTA $^{1}$, KYOKO HIDA ${ }^{2}$, TETSUYA KITAMURA ${ }^{1}$, FUMIHIRO HIGASHINO ${ }^{1}$, \\ NORITAKA OHGA $^{2}$, KAZUAKI FUKUSHIMA ${ }^{3}$ and MASANOBU SHINDOH ${ }^{1}$ \\ Departments of ${ }^{1}$ Oral Pathology and Biology, ${ }^{2}$ Vascular Biology, and ${ }^{3}$ Dental Anesthesiology, \\ Hokkaido University Graduate School of Dental Medicine, Sapporo 060-8586, Japan
}

Received July 20, 2010; Accepted October 13, 2010

DOI: $10.3892 / \mathrm{ol} .2010 .201$

\begin{abstract}
Tumor metastasis to lymph nodes occurs through the lymphatic vessels located in the tumor circumference. However, few studies have focused on the phenotypes of lymphatic vessels around these tumors. We investigated the characteristics of the lymph vessels of tongue squamous cell carcinoma (SCC) and compared them to clinicopathological characteristics. A total of 43 patients diagnosed as having tongue SCC consulted Hokkaido University Hospital were examined. The lymphatic vessels were identified by antibody D2-40 and the number and diameter of tumor lymphatic vessels were measured. The proliferative activity of lymphatic endothelial cells was also examined by immunostaining using antibody MIB-1. We then measured the DNA density of lymphatic endothelial cells in normal and tumor tissues. The number of tumor lymphatic vessels significantly increased in highly metastatic cases of tongue SCC, particularly in cases with a large number of micro lymphatic vessels. A significant correlation was found between the metastatic and proliferative activity of tumor lymphatic endothelial cells. Moreover, the DNA density of tumor lymphatic endothelial cells increased compared to normal tissues. These results suggest that the phenotypes of tumor lymphatic endothelial cells are an indicator of lymph node metastasis of tongue SCC.
\end{abstract}

\section{Introduction}

Lymph node metastasis is a key prognostic indicator for cancer and a guide for deciding the tumor treatment (1-3). In the event of lymph node metastasis, tumor lymphatic vessels are crucial as the tumor cells enter the lymphatic vessels, migrate inside them and settle on the lymph nodes (4). A number of studies were performed on blood vessels (5-7) and the molecular mechanisms involved in the development and maintenance

Correspondence to: Dr Masanobu Shindoh, Department of Oral Pathology and Biology, Hokkaido University Graduate School of Dental Medicine, N13 W7, Kita-ku, Sapporo 060-8586, Japan

E-mail: mshindoh@den.hokudai.ac.jp

Key words: lymphatic vessels, tongue carcinoma, metastasis of blood vessels were largely revealed $(8,9)$. However, lymphangiogenesis and its roles in pathologic conditions have yet to be elucidated.

The peri-tumoral environment is crucial in lymph node metastasis. However, it remains unclear whether lymphatic dissemination is dependent on cancer cell infiltration of newly formed lymphatic vessels that are induced by the tumor or pre-existing lymphatic vessels at peripheral sites of the tumor (10-12).

It was reported that lymphangiogenesis is promoted by lymphatic growth factors derived from tumor cells in a paracrine manner $(4,13,14)$. Therefore, the correlation between the phenotypes of tumor lymphatic vessels and clinicopathological characteristics in tongue squamous cell carcinoma (SCC) was investigated.

\section{Materials and methods}

Patients and tissue samples. A total of 43 samples were obtained from patients who were clinicopathologically diagnosed as having tongue SCC at the Department of Oral Surgery, Hokkaido University Hospital, Japan. Informed consent was obtained from the patients prior to the samples being used. The experiment was conducted under the ethics rules of Hokkaido University Hospital. The clinicopathological characteristics of these patients are shown in Table I.

None of the patients had received pre-operative radiation treatment or chemotherapy. There were 31 male and 12 female patients. The mean age of the patients was 64 years (range 28-91). Tumor size was classified according to the TNM classification of the International Union Against Cancer (UICC). There were $37 \mathrm{~T} 1$ and T2 cases, and $6 \mathrm{~T} 3$ and T4 cases. At the first medical examination, lymph node metastasis $\left(\mathrm{N}^{+} ; \mathrm{N} 1\right.$ and N2) was present in 7 cases. During the follow-up period, subsequent lymph node metastasis (N0 to $\mathrm{N}^{+}$) occurred in 6 of 36 primary N0 patients and distant metastasis in 2 of $43 \mathrm{M} 0$ cases. Lymph node metastasis (primary and subsequent) and distant metastasis are indicative of a poor prognosis. Therefore, the cases that had lymph node metastasis and/or distant organ metastasis were divided into high metastatic potential cases (high-metastatic) and cases without any metastasis as low metastatic potential (low-metastatic). In this study, there were 14 cases of high-metastatic $\left(7 \mathrm{~N}^{+}\right.$, including 
Table I. Clinical and pathological characteristics of the cases examined.

No. of patients

Gender

Male/female

Tumor size

$\mathrm{T} 1 / \mathrm{T} 2$

$\mathrm{T} 3 / \mathrm{T} 4$

Nodal involvement

$\mathrm{N} 0 \rightarrow \mathrm{N}^{-}$

$\mathrm{NO} \rightarrow \mathrm{N}^{+}$

$\mathrm{N}^{+}$

Distant metastasis

$\mathrm{M} 0 \rightarrow \mathrm{M} 1$

Mode of invasion

Expansive

Infiltrative

$1 \mathrm{~N}^{+} \mathrm{M} 0$ to $\mathrm{N}^{+} \mathrm{M} 1,6 \mathrm{~N} 0$ to $\mathrm{N}^{+}$and $1 \mathrm{~N} 0 \mathrm{M} 0$ to M1) and 29 cases of low-metastatic.

Immunohistochemical staining. D2-40 was used to detect peri-tumoral lymphatic vessels. Monoclonal antibody D2-40 was originally raised against M2A, a $40,000 \mathrm{kDa} O$-linked sialoglycoprotein that is expressed in testicular germ cell neoplasias. D2-40 was later shown to react with lymphatic endothelium in formalin-fixed, paraffin-embedded tissues and solid tumors (14).

Serial $4-\mu \mathrm{m}$ sections were cut from formalin-fixed, paraffinembedded tissue blocks, and were then dewaxed and rehydrated through sequential changes of alcohol and distilled water. The slides were boiled in $10 \mathrm{mM}$ sodium citrate buffer ( $\mathrm{pH}$ 6.0) for $3 \mathrm{~min}$ and incubated for $5 \mathrm{~min}$ in $0.3 \%$ hydrogen peroxide, followed by incubation with $1 \% \mathrm{BSA}$ for $10 \mathrm{~min}$. The slides were then incubated with primary antibodies D2-40 (1:50 dilution; Signet Laboratories, Dedham, MA, USA) and MIB-1 (1:100 dilution; Dako, Tokyo, Japan) for $1 \mathrm{~h}$ at room temperature. After being washed with PBS, the sections were incubated with the Histofine Simple Stain PO (M) horseradish peroxidase-conjugated anti-mouse secondary antibody (Nichirei, Tokyo, Japan) for $40 \mathrm{~min}$ and washed in PBS. The color was developed by incubation with the EnVision kit/HRP (DAB; Dako). The slides were then counterstained with hematoxylin and mounted.

\section{Assessment of immunohistochemistry}

Lymphatic vessels in peri-tumoral tissue detected by D2-40. The tumor lymphatic vessels were divided into: micro and large lymphatic vessels, based on diameter. Areas within $500 \mu \mathrm{m}$ from the tumor marginal portion were examined under high-power fields. Lymphatic vessels with diameters $<30 \mu \mathrm{m}$ were defined as micro lymphatic vessels (MLVs) and those with $>30 \mu \mathrm{m}$ as large lymphatic vessels (LLVs). Submucosal lymphatic vessels in normal tissue were also examined as normal counterparts. Three areas were randomly selected and

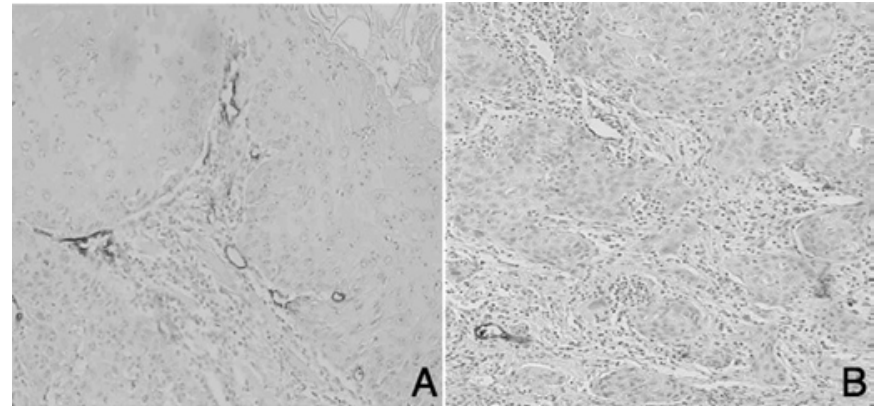

Figure 1. Immunohistochemical detection of microlymphatic vessels (MLVs) by D2-40. (A) Numerous MLVs were noted in the tumor marginal portion in the High-MLV group. (B) Few lymphatic vessels were observed in the Low-MLV group.

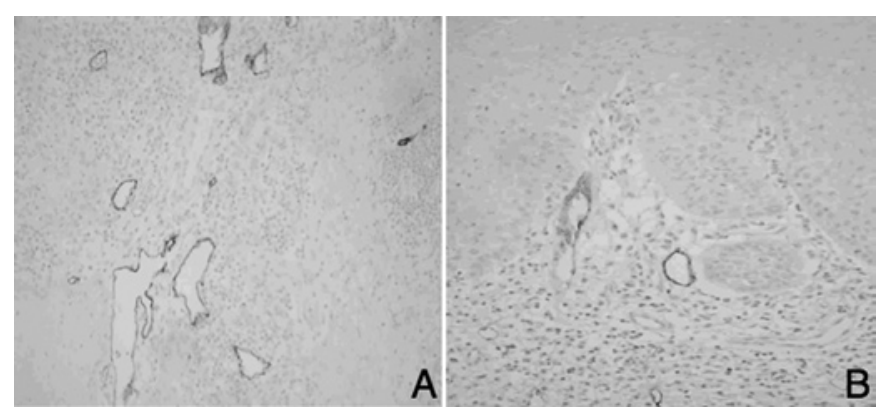

Figure 2. Large lymphatic vessels (LLVs) detected by D2-40. (A) High-LLV vessel group: LLVs were arranged irregularly and located in the tumor marginal portion. (B) The low-LLV group shows the decreased number of vessels.

the number of the two types of vessels was counted. The lesions were divided into four groups: i) High-MLV group, in which the mean number of MLVs was $\geq 5$; ii) Low-MLL group, in which the mean number of MLVs was <5; iii) High-LLV group, in which the mean number of LLVs was $\geq 5$; and iv) Low-LLV group, in which the mean number of LLVs was $<5$.

Proliferative activity of lymphatic endothelial cells. The proliferative activity of lymphatic endothelial cells was examined using antibody MIB-1 in 19 tongue SCC samples from serially-sectioned slides treated with antibody D2-40. Lymphatic vessels with at least one MIB-1-positive lymphatic endothelial cell were defined as lymphatic vessels positive for MIB-1. MIB-1-positive lymphatic vessels were evaluated as proliferating lymphatic vessels and the lymphatic proliferation rate was scored as MIB-1-positive lymphatic vessels/total number of lymphatic vessels (15).

DNA density in tumor lymphatic endothelial cells. The DNA density of lymphatic endothelial cells was examined by measuring the chromatin concentration in 28 cases of tongue SCC. Hematoxylin-stained sections were analyzed by an imaging device (Image Pro Plus). Serial sections were immunostained by MIB-1, and MIB-1-positive endothelial cells were eliminated for scoring in order to select endothelial cells in the resting stage.

Statistical analysis. Correlations between clinicopathological data and states of lymphatic vessels were analyzed using the 
Table II. Relationship between lymphatic vessel characteristics and clinicopathological findings.

\begin{tabular}{|c|c|c|c|c|}
\hline & High-MLV & Low-MLV & High-LLV & Low-LLV \\
\hline \multicolumn{5}{|l|}{ Gender } \\
\hline Male & 15 & 16 & 14 & 17 \\
\hline Female & 5 & 7 & 4 & 8 \\
\hline \multicolumn{5}{|l|}{ Tumor size } \\
\hline $\mathrm{T} 1, \mathrm{~T} 2$ & 16 & 21 & 15 & 22 \\
\hline $\mathrm{T} 3, \mathrm{~T} 4$ & 4 & 2 & 3 & 3 \\
\hline \multicolumn{5}{|c|}{ Tumor metastatic potential } \\
\hline High-metastatic & 11 & 3 & 11 & 3 \\
\hline Low-metastatic & 9 & 20 & 7 & 22 \\
\hline \multicolumn{5}{|l|}{ Mode of invasion } \\
\hline Expansive & 5 & 12 & 3 & 14 \\
\hline Infiltrative & 15 & 11 & 15 & 11 \\
\hline
\end{tabular}

$\chi^{2}$ test. The relationships between lymphatic proliferation and the tumor metastatic potential, as well as between the nuclear DNA density of tumor lymphatic endothelial cells and clinicopathological characteristics were tested using the unpaired t-test and Mann-Whitney U test.

\section{Results}

Micro and large lymphatic vessels and clinicopathological factors. D2-40-positive lymphatic vessels in tongue SCC are shown in Fig. 1. Numerous MLVs were located adjacent to the tumor marginal portion in the high-MLV group (High-MLV, Fig. 1A), whereas few lymphatic vessels were observed in the low-MLV group (Low-MLV, Fig. 1B). A total of 20 cases of High-MLV and 23 of Low-MLV were noted. LLVs were arranged irregularly and were located at a distance from the tumor marginal portion than that of MLVs. There were 18 cases of High-LLV (Fig. 2A) and 25 cases of Low-LLV (Fig. 2B).

No correlation was found between the characteristics of the lymphatic vessels and age, gender or $\mathrm{T}$ stage. On the other hand, a highly significant correlation was found between cases with high-metastatic and High-MLV $\left(\mathrm{P}=0.0034, \chi^{2}\right.$ test $)$ and High-LLV groups $\left(\mathrm{P}=0.00069, \chi^{2}\right.$ test) (Table II, Fig. 3 ). In addition, an increasing tendency of the infiltrating mode of invasion and High-MLV $(\mathrm{P}=0.069)$ and a significant correlation between cases with an infiltrating invasion pattern of cancer and High-LLV ( $\mathrm{P}=0.00925)$ were observed (Fig. 4).

Proliferative activity of lymphatic endothelial cells and tumor metastasis. MIB-1 immunostaining was performed for 19 cases of tongue SCC, using serial sections stained with D2-40. Lymphatic vessels with at least one MIB-1-positive lymphatic endothelial cell were defined as MIB-1-positive lymphatic vessels and tumor lymphatic endothelial cell proliferation was investigated.

The identification of MIB-1-positive lymphatic vessels in the normal tissue samples proved difficult. However, MIB-1positive lymphatic vessels were increased around the tumor area of tongue SCC. D2-40-positive lymphatic endothelial cells (Fig. 5A) and MIB-1 expression of lymphatic endothelial cells (Fig. 5B) is shown in the serial sections.

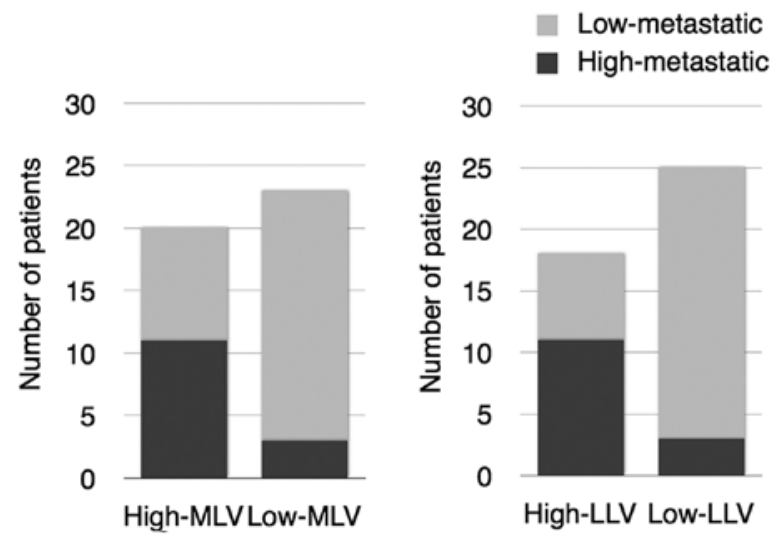

Figure 3. A highly significant correlation between high-metastatic cases and both High-MLV and High-LLV groups $\left(\mathrm{P}<0.01, \chi^{2}\right.$ test $)$.

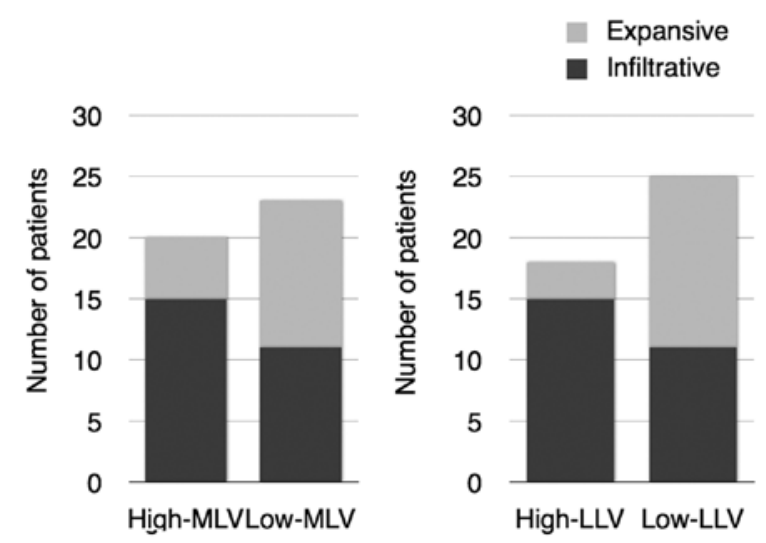

Figure 4. High-MLV and -LLV groups exhibited a correlation with tumor infiltrative growth.

MLV and LLV exhibited MIB-1-positive lymphatic endothelial cells. High-metastatic cases had a higher ratio of MIB-1-positive MLVs (23.6\%). By contrast, the mean percentage of proliferating lymphatic vessels was $8 \%$ in the low-metastatic cases (unpaired one-tailed t-test, $\mathrm{P}<0.05$, 


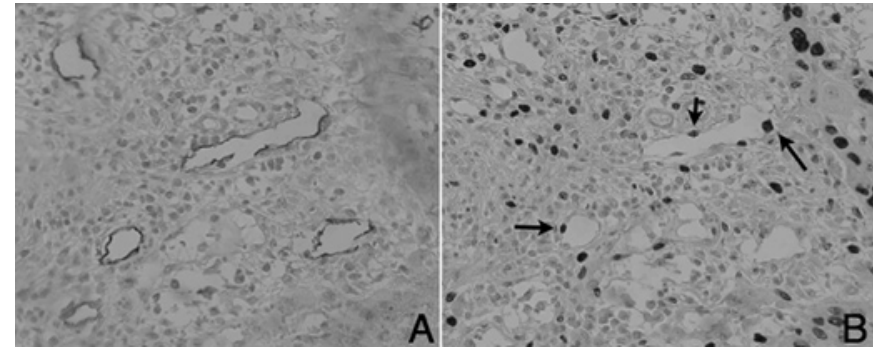

Figure 5. MIB-1 expression in tumor lymphatic endothelial cells in serial sections. (A) D2-40-positive MLVs. (B) MIB-1 expression is observed in the nucleus of lymphatic endothelial cells (indicated by the arrows).

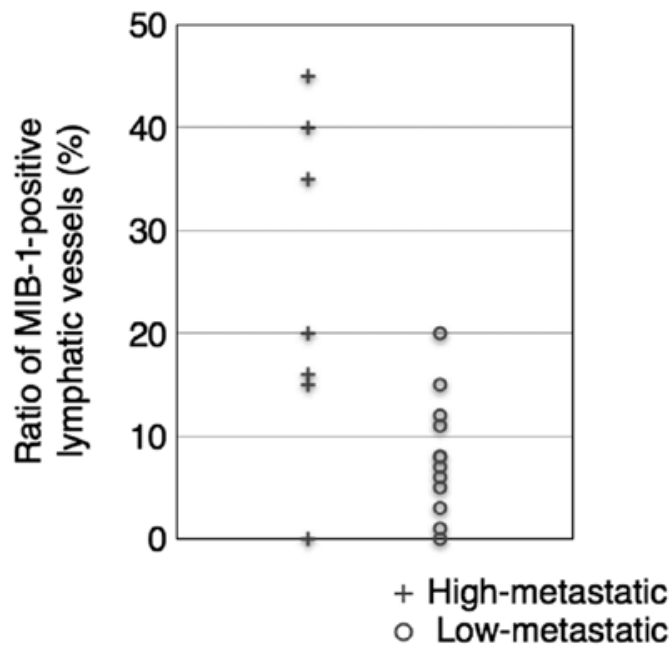

Figure 6. Lymphatic endothelial cells were noted to be actively proliferating in the High-metastatic cases $(\mathrm{P}<0.05)$.

Fig. 6). Thus, lymphatic endothelial cells were noted to be actively proliferating in the high-metastatic cases.

DNA contents of tumor lymphatic endothelial cells. The nuclear DNA density of tumor lymphatic vessels was estimated in 28 cases. The tumor endothelial cells had a significant higher nuclear DNA density compared to the lymphatic endothelial cells in normal tissue. A higher correlation was observed between the nuclear DNA density of tumor lymphatic endothelial cells and the metastatic potential. The mean DNA density of endothelial cells in the low-metastatic cases was 1.17-fold higher than that in normal tissue, and 1.38-fold higher in the high-metastatic cases, which was statistically significant (two-tailed Mann-Whitney U test, P<0.01, Fig. 7).

\section{Discussion}

Tumor lymphatic vessels were shown to be induced by vascular endothelial cell growth factors (VEGFs) that are secreted by tumor cells, and this induction is thought to promote lymph node metastasis $(1,4,16,17)$. Lymphatic vessels are classified into initial and collecting lymphatic vessels (18). Morphological differences between initial and collecting lymphatic vessels include the size of the vessels and the existence of smooth muscle cells and intraluminal valves. Initial lymphatic vessels are smaller and have primary valves

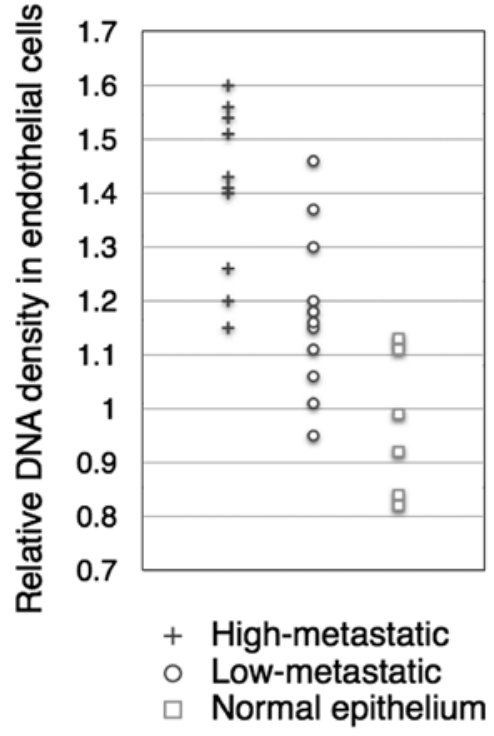

Figure 7. A significant correlation between the nuclear DNA density of tumor lymphatic endothelial cells and the metastatic potential. The tumor lymphatic endothelial cells exhibited a higher nuclear DNA density than the normal endothelial cells and the tumor lymphatic endothelial cells in the High-metastatic cases exhibited a higher nuclear DNA density than the Lowmetastatic cases and the normal counterparts $(\mathrm{P}<0.01)$.

that are composed of partial overlaps between lymphatic endothelial cells that are involved in the activity of lymphatic vessels $(18,19)$. Padera et al $(20)$ reported that newly formed small intratumoral lymphatic vessels did not have any correlation with lymphatic metastasis since intratumoral lymphatic vessels were not functional and enlarged lymphatic vessels were correlated with tumor metastasis. On the other hand, Skobe et al (21) reported that breast cancer cells secreted VEGF-C, which increased intratumoral lymphangiogenesis and resulted in significantly enhanced metastasis to the regional lymph nodes and lungs. Thus, the significance of the lymphatic vessels around tumors remains to be determined.

We examined the correlation between the phenotypes of tumor lymphatic vessels and the clinicopathological characteristics in tongue SCC. Highly significant metastatic potential was observed in cases in the High-MLV and -LLV groups. Skobe et al transplanted VEGF-C-overexpressing breast cancer cell lines into nude mice and found numerous small lymphatic vessels in the stromal tissue of transplanted VEGF-C-overexpressing MDA-MB-435 cells. These authors also reported that VEGF-C-overexpressing tumors were infiltrated throughout the central tumor areas by lymphatic vessels, whereas control tumors were not invaded by lymphatic vessels (21). Our results were in agreement with the report (15) that tumor MLVs may be regarded as newly formed lymphatic vessels, indicating the ability for tumor lymphangiogenesis. Our results suggest that lymph node metastasis of tongue SCC is predicted by estimating lymphatic vessels around the tumor, even in the early stages of tumor progression.

In the High-MLV and -LLV groups, a correlation to the infiltrative growth pattern of tumors was noted. Ueda et al (16) reported that VEGF-C-expressing ovarian carcinoma cell lines showed an invasive phenotype with correlated expression levels of VEGF-C and MMP-2. The precise mechanisms of 
VEGF-C and MMP-2 expression have yet to be elucidated, but it may be postulated that cases in the high tumor lymphatic vessel group express a high level of VEGF-C as well as MMP-2 or other extracellular matrix-degrading enzymes that may be involved in invasive potential.

We examined the proliferative activity of tumor lymphatic vessels by immunostaining serial sections with MIB-1 and D2-40 antibodies. In 19 cases of tongue SCC, both MLV and LLV had a greater number of MIB-1-positive lymphatic endothelial cells compared to lymphatic vessels in normal tissue. Moreover, the high-metastatic group showed an increasing ratio of MIB-1-positive endothelial cells. These results suggested that newly formed lymphatic vessels were closely associated with regional lymph node metastases.

It is known that the nuclear DNA contents of tumor cells increase due to chromosome abnormalities (22). Malignant tumor cells are known to have a broad range of nuclear DNA content compared to normal or benign neoplastic cells. However, stromal cells in cancer are considered to be intact, including endothelial cells in epithelial tumors. Hida et al reported that tumor vascular endothelial cells exhibit genetic abnormalities (23-25).

We measured the DNA content of tumor lymphatic vessels using an imaging device. The nuclear DNA content was found to increase in tumor lymphatic compared to normal lymphatic endothelial cells. Cases in the high-metastatic group showed an increased DNA content in tumor lymphatic endothelial cells compared to those in the low-metastatic cells. Our results indicate that tumor lymphatic endothelial cells may have genetic abnormalities simialr to those observed in tumor vascular endothelial cells. Further studies are required to determine the characteristics of tumor lymphatic endothelial cells and the inhibitory mechanism of lymph node metastasis, which may contribute to the welfare of patients diagnosed with tongue SCC.

\section{Acknowledgements}

This study was supported in part by Grants-in-Aid for Scientific Research from the Ministry of Education, Culture, Sports, Science and Technology of Japan.

\section{References}

1. He Y, Karpanen $\mathrm{T}$ and Alitalo K: Role of lymphangiogenic factors in tumor metastasis. Biochim Biophys Acta 1654: 3-12, 2004.

2. Achen MG, McColl BK and Stacker SA: Focus on lymphangiogenesis in tumor metastasis. Cancer Cell 7: 121-127, 2005.

3. Bird-Lieberman EL and Fitzgerald RC: Early diagnosis of oesophageal cancer. Br J Cancer 101: 1-6, 2009.
4. Karpanen T, Egeblad M, Karkkainen MJ, et al: Vascular endothelial growth factor $\mathrm{C}$ promotes tumor lymphangiogenesis and intralymphatic tumor growth. Cancer Res 61: 1786-1790, 2001.

5. Nor JE and Polverini PJ: Role of endothelial cell survival and death signals in angiogenesis. Angiogenesis 3: 101-116, 1993.

6. Moldovan NI and Asahara T: Role of blood mononuclear cells in recanalization and vascularization of thrombi: past, present, and future. Trends Cardiovasc Med 13: 265-269, 2003.

7. Blagosklonny MV: Antiangiogenic therapy and tumor progression. Cancer Cell 5: 13-17, 2004.

8. Coultas L, Chawengsaksophak K and Rossant J: Endothelial cells and VEGF in vascular development. Nature 438: 937-945, 2005.

9. Stoeltzing O, Meric-Bernstam F and Ellis LM: Intracellular signaling in tumor and endothelial cells: the expected and, yet again, the unexpected. Cancer Cell 10: 89-91, 2006.

10. Breiteneder-Geleff S, Soleiman A, Kowalski $\mathrm{H}$, et al: Angiosarcomas express mixed endothelial phenotypes of blood and lymphatic capillaries: podoplanin as a specific marker for lymphatic endothelium. Am J Pathol 154: 385-394, 1999.

11. Oliver G: Lymphatic vasculature development. Nat Rev Immunol 4: 35-45, 2004.

12. Akishima Y, Ito $\mathrm{K}$, Zhang L, et al: Immunohistochemical detection of human small lymphatic vessels under normal and pathological conditions using the LYVE-1 antibody. Virchows Arch 444: 153-157, 2004

13. Pepper MS and Skobe M: Lymphatic endothelium: morphological, molecular and functional properties. J Cell Biol 163 209-213, 2003.

14. Fukunaga M: Expression of D2-40 in lymphatic endothelium of normal tissues and in vascular tumours. Histopathology 46: 396-402, 2005

15. Koukourakis MI, Giatromanolaki A, Sivridis E, et al: LYVE-1 immunohistochemical assessment of lymphangiogenesis in endometrial and lung cancer. J Clin Pathol 58: 202-206, 2005.

16. Ueda M, Hung YC, Terai Y, et al: Vascular endothelial growth factor-C expression and invasive phenotype in ovarian carcinomas. Clin Cancer Res 11: 3225-3232, 2005.

17. Aishima S, Nishihara Y, Iguchi T, et al: Lymphatic spread is related to VEGF-C expression and D2-40-positive myofibroblasts in intrahepatic cholangiocarcinoma. Mod Pathol 21: 256-264, 2008.

18. Hagendoorn J, Padera TP, Kashiwagi S, et al: Endothelial nitric oxide synthase regulates microlymphatic flow via collecting lymphatics. Circ Res 95: 204-209, 2004.

19. Hagendoorn J, Padera TP, Fukumura D and Jain RK: Molecular regulation of microlymphatic formation and function: role of nitric oxide. Trends Cardiovasc Med 15: 169-173, 2005.

20. Padera TP, Kadambi A, di Tomaso E, et al: Lymphatic metastasis in the absence of functional intratumor lymphatics. Science 296: 1883-1886, 2002.

21. Skobe M, Hawighorst T, Jackson DG, et al: Induction of tumor lymphangiogenesis by VEGF-C promotes breast cancer metastasis. Nat Med 7: 192-198, 2001.

22. Rubio Bueno P, Naval Gias L, Garcia Delgado R, et al: Tumor DNA content as a prognostic indicator in squamous cell carcinoma of the oral cavity and tongue base. Head Neck 20: 232-239, 1998.

23. Hida K, Hida Y, Amin DN, et al: Tumor-associated endothelial cells with cytogenetic abnormalities. Cancer Res 64: 8249-8255, 2004.

24. Hida K, Hida Y and Shindoh M: Understanding tumor endothelial cell abnormalities to develop ideal anti-angiogenic therapies. Cancer Sci 99: 459-466, 2008.

25. Akino T, Hida K, Hida Y, et al: Cytogenetic abnormalities of tumor-associated endothelial cells in human malignant tumors. Am J Pathol 175: 2657-2667, 2009. 